\title{
THE INFLUENCE OF ALTITUDE TRAINING ON SELECTED BLOOD PARAMETERS AND LACTATE CURVES DURING SWIMMING
}

\author{
Boro Štrumbelj ${ }^{(\mathrm{A}, \mathrm{B}, \mathrm{C}, \mathrm{D}, \mathrm{E}, \mathrm{F})}$, Anton Ušaj $^{(\mathrm{F}, \mathrm{G})}$ \\ Laboratory of Biodynamics, Faculty of Sport, University of Ljubljana, Ljubljana, Slovenia
}

\begin{abstract}
Introduction: Moderate altitude training has become popular to improve competition performance in swimming both at altitude and sea level. The aim of the present study was to examine the influence of moderate altitude training on lactate curve during three weeks and after the exposure to altitude training at $1860 \mathrm{~m}$ and to examine the effects of altitude training on selected blood parameters before and after the training at altitude.

Methods: In the study participated four (1 men, 3 women) high competition-level swimmers (mean \pm SD; age $21 \pm 1.2$ years). All swimmers performed 5 times standardized test $5 \times 3 \times 200 \mathrm{~m}$ to obtain velocities and heart rates at lactate thresholds and OBLA thresholds (before, three times on altitude and after altitude exposure). Blood samples for selected blood parameters (Erci, $\mathrm{Hb}, \mathrm{Ht}$, ERC-ret and ferritin) were taken before and after exposure to altitude.

Results: There were no significant changes $(P<0.05)$ in heart rate and blood lactate at a lactate threshold and OBLA threshold during altitude camp and 21 days after return at sea level. Individual response at altitude camp were found above OBLA threshold. No significant differences were found in selected blood parameters before and after exposure to altitude training (Erci, Hb, Ht, ERC-ret and ferritin).

Conclusions: These data indicate that no significant changes in lactate threshold, OBLA threshold, and selected blood parameters were found at altitude and upon return to sea-level after three weeks of training at $1860 \mathrm{~m}$.
\end{abstract}

Key words: swimming, moderate altitude, lactate threshold, OBLA threshold, blood

\section{Introduction}

Moderate altitude training (approximately 1800 to $2600 \mathrm{~m}$ ) has become popular to improve competition performance in swimming both at altitude and sea level. When swimmers are exposed acutely to moderate altitude, a number of physiological responses occur that can comprise performance at altitude because exposure results in a reduction in partial pressure of oxygen in the arterial blood and a reduction in oxygen content; these include increased ventilation, increased heart rate, decreased stroke volume, reduced plasma volume, and lower maximal aerobic power [1]. In an attempt to maintain aerobic metabolism during increased effort, a series of acclimatization responses occur. Among the most conspicuous of these responses is an increase in hemoglobin $(\mathrm{Hb})$ concentration. The increase in $\mathrm{Hb}$ has been construed as the fundamental adaptation enabling increases in aerobic power and performance to occur on return to sea-level $[2,3]$, thus an improved oxygen carrying capacity depending on the adequate hypoxic dose. There has been extensive research into the effects of altitude sojourn on physiological capacities on volunteer (moderate- to well-trained) subjects and exercise performance at sea level $[1,4]$. However, relatively few studies have monitored elite swimmers during a competitive racing season with altitude sojourn incorporated into their training [5]. The aim of the present study was to examine the influence of moderate altitude training on lactate curve during three weeks and after the exposure to altitude training at $1860 \mathrm{~m}(\mathrm{La}$ Ioma, Mexico). We also examined the effects of altitude training on selected blood parameters before and after the training at altitude.

\section{Materials and Methods: \\ Subjects}

Four elite swimmers ( 1 male; 4 female) voluntarily participated in this study. Subject characteristics were as follows: age $21.0 \pm 1.2 \mathrm{y}$, mean $\pm \mathrm{SD}$; body mass 66.1 $\pm 9 \mathrm{~kg}$. All swimmers were competing on Olympic games in Bejing. The subjects were informed of the experimental procedures and possible risks involved with participation before written consent was obtained.

\section{Experimental Protocol}

All swimmers performed 5 times a standardized lactate profile/ «step test « $5 \times 3 \times 200 \mathrm{~m}$ starting at an intensity of about $80 \%$ of personal best performance at the time of testing. Intensity was increased by four second per step. The rest period was 30 second during single set and 3 minutes during series. Lactate curves and velocities and heart rates at lactate thresholds and OBLA thresholds were obtained with use of computer module in GFA Basic. Lactate test were performed 5 
days before altitude sojourn, three times on altitude (on $6^{\text {th }}$ day, $13^{\text {th }}$ day and $20^{\text {th }}$ day) and 21 days after altitude sojourn. Capillary blood samples $(60-80 \mu \mathrm{l})$ were taken by micro puncture from the hyperemic earlobe. Blood samples for measuring [ $\left.\mathrm{LA}^{-}\right]$were diluted in a LKM41 lactate solution (LANGE, Germany) and analyzed using the MINI8 (LANGE, Germany) photometer. The heart rate was recorded using Polar heart rate monitors. Lactate thresholds and OBLA thresholds were obtained with use of computer program GFA basic.

Blood samples $(5 \mathrm{ml})$ were drawn from an antecubital vein in the sitting position for analysis before and after exposure to altitude. A local hematology laboratory (Clinical center, Ljubljana) analyzed venous blood samples for erythrocytes, hemoglobin, hematocryt, reticulocytes and ferritin concentration via automated instrumentation.

\section{Training}

The team trained 20 days with two full day off (day 4 and 18). The training load consisted of 35 water sessions (two in one day) and 10 "gym" sessions. The total volume for camp was 205,800 meters. The athletes performed mainly endurance training with intensities below a lactate level of $4 \mathrm{mmol} \cdot \mathrm{l}^{-1}$.

\section{Statistical analysis}

The values are presented as means \pm standard deviations $(\mathrm{SD})$. Individual one-way repeated measures
ANOVAs were employed to test for any significant differences between velocities and heart rates at lactate thresholds and OBLA thresholds followed by Dunnett's test for multiple comparison procedure to determine source of difference. A Student's paired $t$-test was used to compare the hematological data between pre- and post-altitude sojourn. A 95\% level of confidence was accepted for all comparisons. All statistical parameters were calculated using SPSS (version "Version 10.0"). Significance was considered with $P<0.05$.

\section{Results}

Lactate thresholds, OBLA thresholds, heart rates at thresholds and individual lactate curves and heart rates response during step test

No significant differences were found in both velocities thresholds (lactate threshold and OBLA threshold). Heart rate values on thresholds velocities were also unchanged.

From comparisons of individual lactate curves for subject S.I. is visible that no evident right or left shift of the lactate curve was found. The most pronounced difference was found for the lactate curve above the OBLA thresholds where the slope of lactate curve was more pronounced during altitude exposure. Heart rate values shifted slightly to the right after the first test however not markedly.

From comparisons of individual lactate curves for subject L.T. is visible that evident left shift of the lactate curve was found during altitude exposure. From the

Table 1. Average velocities and heart rates at lactate thresholds and OBLA thresholds for all swimmers (N.S. difference; $P<0.05)$

\begin{tabular}{|l|c|c|c|c|c|}
\hline & Before & 1 . Altitude & 2. Altitude & 3. Altitude & After \\
\hline $\mathrm{V}_{\mathrm{LT}}\left(\mathrm{m} \cdot \mathrm{s}^{-1}\right)$ & $1.38 \pm 0.09$ & $1.38 \pm 0.06$ & $1.37 \pm 0.06$ & $1.38 \pm 0.06$ & $1.41 \pm 0.09$ \\
\hline$[\mathrm{LA}]_{\mathrm{LT}}$ & $1.6 \pm 0,3$ & $1.5 \pm 0,5$ & $1.4 \pm 0.2$ & $1.4 \pm 0,4$ & $1.6 \pm 0,3$ \\
\hline $\mathrm{HR}_{\mathrm{LT}}\left(\mathrm{b} \cdot \mathrm{min}^{-1}\right)$ & $161.5 \pm 7$ & $161.7 \pm 4$ & $159.5 \pm 4$ & $159.5 \pm 7$ & $164.2 \pm 4$ \\
\hline $\mathrm{V}_{\mathrm{OBLA}}\left(\mathrm{m} \cdot \mathrm{s}^{-1}\right)$ & $1.43 \pm 0.09$ & $1.42 \pm 0.06$ & $1.41 \pm 0.07$ & $1.42 \pm 0.06$ & $1.45 \pm 0.09$ \\
\hline $\mathrm{HR}_{\mathrm{OBLA}}\left(\mathrm{b} \cdot \mathrm{min}^{-1}\right)$ & $171.0 \pm 7$ & $172.5 \pm 2$ & $172.00 \pm 4$ & $172.0 \pm 6$ & $173.7 \pm 3$ \\
\hline
\end{tabular}
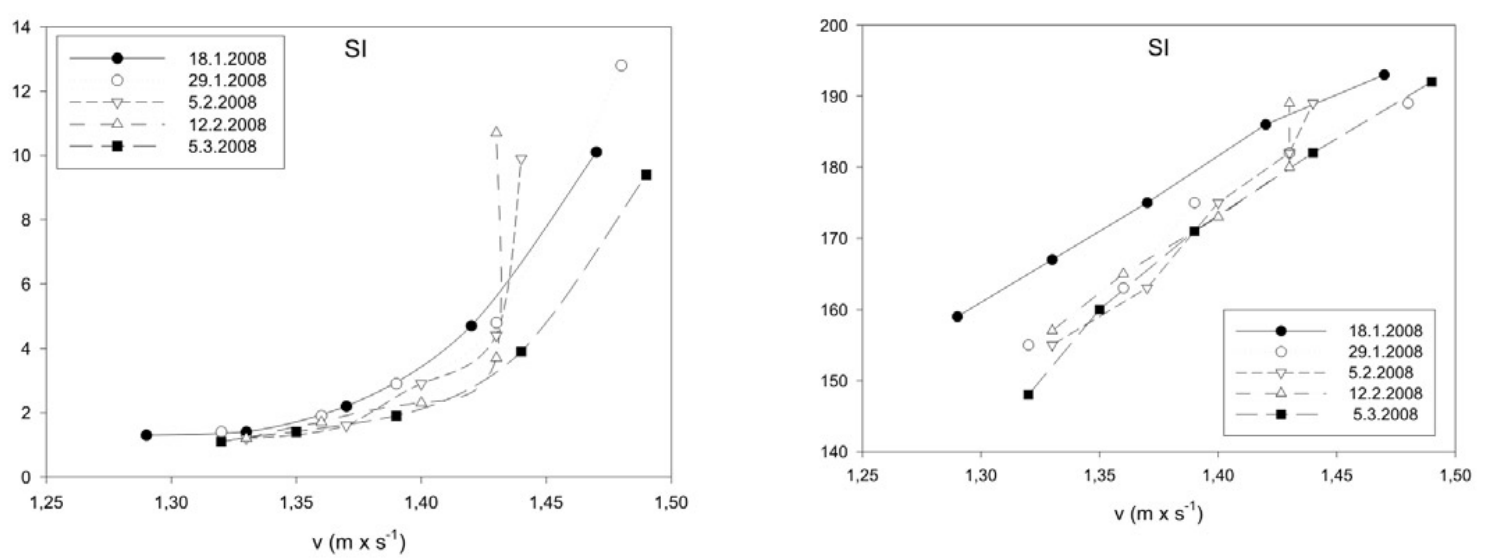

Figure 1. Individual lactate curves and heart rates response during a standardized lactate profile/«step test «5x3x200m before, during and after altitude camp for subject S.I. 

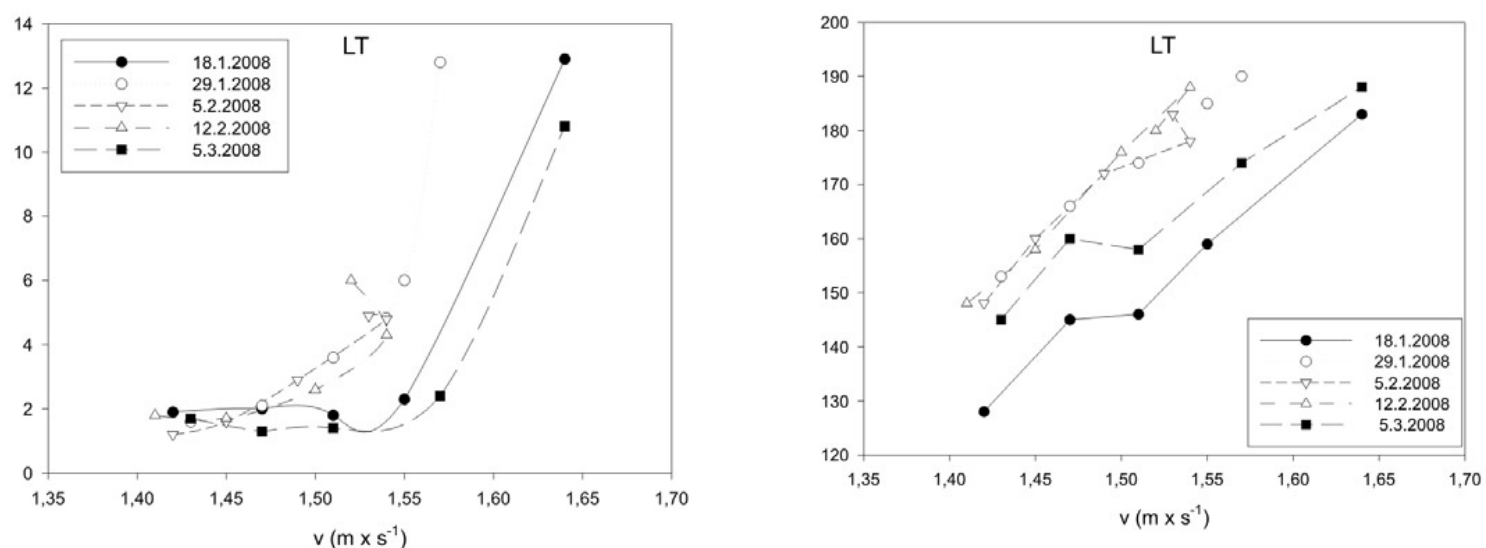

Figure 2. Individual lactate curves and heart rates response during a standardized lactate profile/«step test « $5 \times 3 \times 200 \mathrm{~m}$ before, during and after altitude camp for subject L.T.
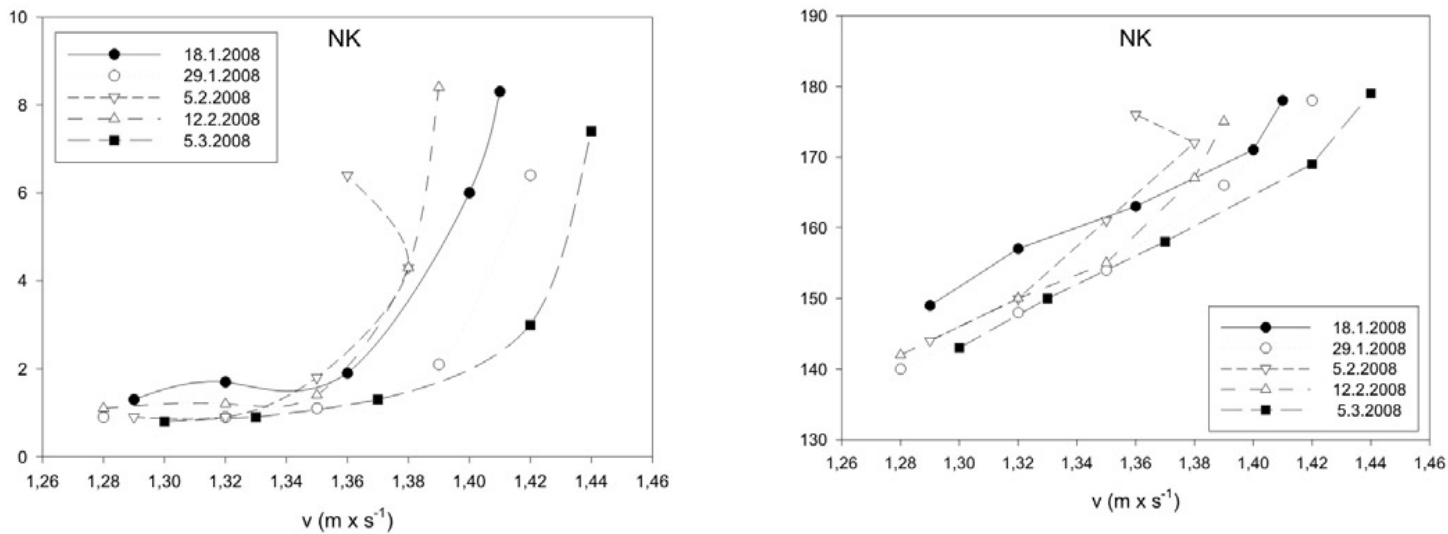

Figure 3. Individual lactate curves and heart rates response during a standardized lactate profile/«step test $4 \times 3 \times 200 \mathrm{~m}$ before, during and after altitude camp for subject N.K.
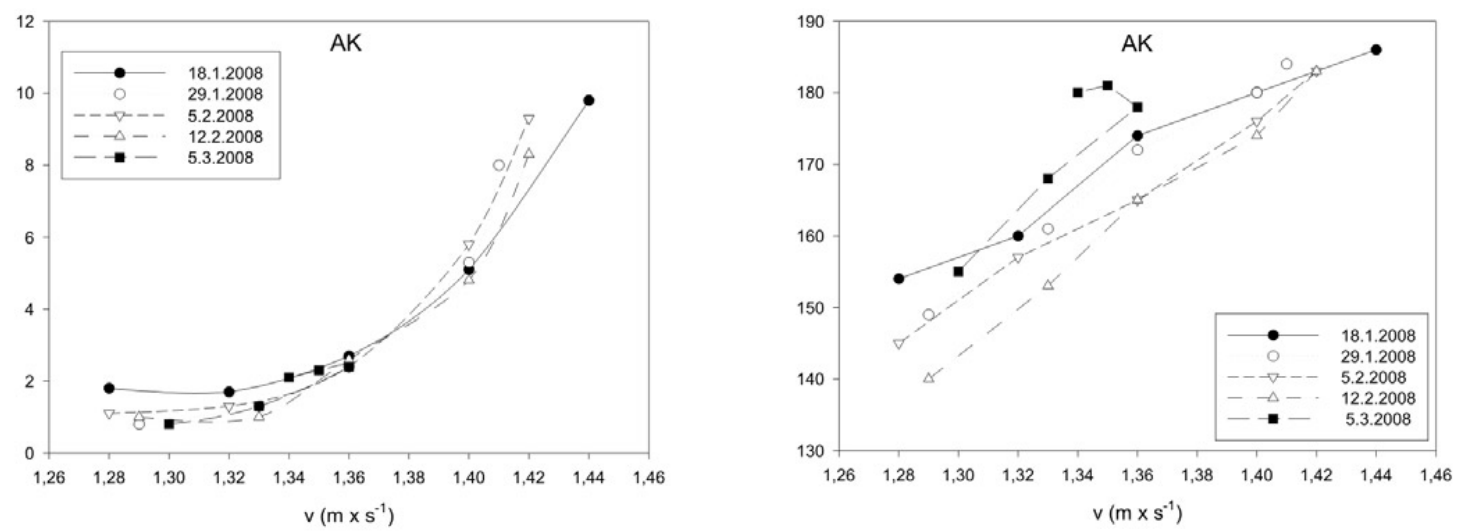

Figure 4. Individual lactate curves and heart rates response during a standardized lactate profile/«step test « $5 \times 3 \times 200 \mathrm{~m}$ before, during and after altitude camp for subject A.K.

lactate curves obtained during second and third test at the altitude lower maximal obtained lactate values are also visible. Heart rate values shifted slightly to the left during all three tests at altitude. After the altitude camp a shift of heart rate curve to the right is visible on sea level.

From comparisons of individual lactate curves for subject N.K. is visible that evident left shift of the lactate curve was found during altitude exposure only for second and third lactate test. First test at altitude exposure resulted in right shift of the lactate curve. All changes were more pronounced above lactate thresholds. Heart rate values during all four tests were found at altitude in between first and second test at sea level. After the altitude camp a shift of lactate curve to the right was visible above lactate threshold.

From comparisons of individual lactate curves and obtained thresholds for subject A.K. is visible that no evident left or right shift of the lactate curve were found during altitude exposure. Only last lactate curve 
on sea level resulted in lowered maximal lactate values and higher heart rates in comparison to all other tests probably due to exhaustion of the swimmer.

\section{Erythrocytes, hemoglobin, hematocrit, reticulocytes and ferritin before and after altitude camp}

Table 2. Absolute changes of erythrocytes, hematocrit, hemoglobin, reticulocytes and ferritin before and after altitude camp (N.S. difference; $P<0.05$ )

\begin{tabular}{|l|c|c|}
\hline & Before & After \\
\hline $\mathrm{RBC}\left(10^{12} \cdot \mathrm{l}^{-1}\right)$ & $4.82 \pm 0.27$ & $4.71 \pm 0.35$ \\
\hline $\mathrm{Hb}\left(\mathrm{g} \cdot \mathrm{l}^{-1}\right)$ & $143.50 \pm 9.26$ & $143.25 \pm 12.45$ \\
\hline $\mathrm{Hct}(1)$ & $0.44 \pm 0.03$ & $0.43 \pm 0.03$ \\
\hline ERC- RET $(\%)$ & $1.35 \pm 0.54$ & $1.65 \pm 1.05$ \\
\hline Ferritin $\left(\mathrm{ng} \cdot \mathrm{ml}^{-1}\right)$ & $58 \pm 14.72$ & $44.25 \pm 23.91$ \\
\hline
\end{tabular}

Although reticulocytes increased from $1.35 \pm 0.54 \%$ to $1.65 \pm 1.05 \%$ and ferritin decreased from $58 \pm 14.72$ ng. $\mathrm{ml}^{-1}$ to $44.25 \pm 23.91 \mathrm{ng} \cdot \mathrm{ml}^{-1}$ no significant differences were found in selected blood parameters before and after exposure to altitude training.

The data revealed inter-individual variability of all selected blood parameters were pre- to post-altitude measurements were analysed where no significant difference were found (Table 2. and 3.).

\section{Discussion}

Selected training on moderate altitude surprisingly didn't have influence on lactate thresholds and OBLA thresholds during three weeks of exposure to altitude, neither after the altitude exposure. We found no evident shift of lactate curve to the left on the altitude camp below OBLA threshold because altered oxygen supply. However we found large inter-individual variations in the shifts of lactate curve and heart rate response during incremental test after arrival and during altitude sojourn, especially above OBLA threshold. This inter-individual variations and small number of subjects influenced on our results. However from our results is clearly visible that coaches should take into consideration individual response to altitude sojourn when training intensity is planned. From our results is also visible that lactate curve is more affected by altitude above OBLA threshold and consequently anaerobic capacity can also be influenced by altitude training. This has been shown also in other study [6].

One of the principal observation of this study is that a 21 -day sojourn to moderate altitude $(1860 \mathrm{~m})$ does not lead to a significant increase of erythrocytes, hemoglobin, hematocrit, reticulocytes or decrease of ferritin. Hypoxia-induced secondary polycythemia is a major contributor to increased work capacity at altitude. The common finding upon exposure to hypoxia is a transient increase in hemoglobin concentration and hematocrit because of a rapid decrease in plasma volume followed by an increase in erythropoiesis per se. Both nonathletes and elite endurance athletes have maximal reticulocytosis after about 8 to 10 days at moderate altitude. Training periods of 3 weeks at moderate altitudes result in individual increase of hemoglobin concentration of about 1 to $4 \%$. A more accentuated increase in hemoglobin can be obtained with longer sojourns at moderate altitude. The most important 'erythropoiesis-specific' nutrition factor is iron availability which can modulate erythropoiesis over a wide range in humans. Adequate iron stores are a necessity for haematological adaptation to hypoxia. However, at moderate altitude, there is a need for rapid mobilization of iron and even if the stores are normal there is a risk that they cannot be mobilized fast enough for an optimal synthesis of hemoglobin. Data from healthy athletes training at moderate altitudes suggest a true increase in hemoglobin concentration of about $1 \%$ per week. Complete haematological adaptation occurred when sea level residents have similar hemoglobin concentrations at moderate altitude compared with residents. This difference indicates a necessary adaptation time of about 12 weeks. If the training period at moderate altitude must be shorter, several sojourns at short intervals are recommended [7]. Our results are in agreement with previous investigations of moderate altitude on erythropoesis such as the study of Pottgiesser et al. [8], Friedmann et al. [5] and Saunders et al. [4]. The results of our study support the hypothesis that altitudes above $2100 \mathrm{~m}$ might be considered as a

Table 3. Absolute individual changes of erythrocytes, hemoglobin, hematocrit, reticulocytes and ferritin before and after altitude camp

\begin{tabular}{|l|c|c|c|c|c|c|c|c|}
\hline \multirow{2}{*}{} & \multicolumn{2}{|c|}{ S.I. } & \multicolumn{2}{c|}{ K.A. } & \multicolumn{2}{c|}{ N.K. } & \multicolumn{2}{c|}{ L.T. } \\
\cline { 2 - 10 } & $\mathrm{B}$ & $\mathrm{A}$ & $\mathrm{B}$ & $\mathrm{A}$ & $\mathrm{B}$ & $\mathrm{A}$ & $\mathrm{B}$ & $\mathrm{A}$ \\
\hline $\mathrm{RBC}\left(10^{12} \cdot \mathrm{1}^{-1}\right)$ & 4,91 & 4,83 & 4,72 & 4,83 & 4,5 & 4,19 & 5,14 & 4,97 \\
\hline $\mathrm{Hb}\left(\mathrm{g} \cdot \mathrm{l}^{-1}\right)$ & 141 & 140 & 140 & 145 & 136 & 129 & 157 & 159 \\
\hline $\mathrm{Hct}(1)$ & 0,445 & 0,435 & 0,42 & 0,434 & 0,416 & 0,392 & 0,476 & 0,457 \\
\hline ERC- RET $(\%)$ & 1 & 1,1 & 1,4 & 1,4 & 2,1 & 3,2 & 0,9 & 0,9 \\
\hline Ferritin $\left(\mathrm{ng} \cdot \mathrm{ml}^{-1}\right)$ & 57 & 64 & 59 & 43 & 40 & 11 & 76 & 59 \\
\hline
\end{tabular}


threshold for enhanced erythropoesis by elite athletes [9]. We found also inter-individual variations of erythropoetic adaptations found also by Chapman et al. [10] and Friedmann et al. [5].

\section{Conclusions}

Selected training on moderate altitude surprisingly didn't have influence on lactate thresholds and OBLA thresholds during three weeks of sojourn at altitude, neither after the altitude sojourn. Selected blood parameters which influence oxygen transport capacity of blood were also unchanged after altitude sojourn in comparison to sea levels before the altitude training. However it should be taken into consideration that inter-individual variations were found and also a small number of subjects influenced on our results.

\section{References:}

1. Bailey DM, Davies B. Physiological implications of altitude training for endurance performance at sea level: a review. $\mathrm{Br}$ J Sports Med 1997; 31:183-90.

2. Green H.J. Altitude acclimatization, training and performance. J Sci Med Sport 2000; 3(3): 299-312.

3. Levine BD, Stray-Gundersen J. A practical approach to altitude training: where to live and train for optimal performance enhancement. Int J Sports Med 1992; 13(Suppl 1): S209-12.

4. Saunders PU, Telford RD, Pyne DB, et al. Improved running economy and increased hemoglobin mass in elite runners after extended moderate altitude exposure, J Sci Med Sport 2009;12(1): 67-72.
5. Friedmann B., Frese F, Menold E etr al. Individual variation in the erythropoietic response to altitude training in elite junior swimmers. Br J Sports Med 2005; 39:148-53.

6. Svedenhag J, Saltin B, Johanson C, et al. Aerobic and anaerobic exercise capacities of elite middle-distance runners after two weeks of training at moderate altitude. Scand J Med Sci Sports 1991; 1:205-14.

7. Berglund B. High altitude training. Aspects of haematological adaptation. Sports Med 1992; 14: 289-303.

8. Pottgiesser T, Ahlgrim C, Ruthardt $\mathrm{S}$ et al. Hemoglobin mass after 21 days of conventional altitude training at $1816 \mathrm{~m}$. J Sci Med Sport 2009; 12(6): 673-5.

9. Rusko HK, Tikkanen HO, Peltonen JE. Altitude and endurance training. J Sports Sci 2004; 22(100): 928-44.

10. Chapman RF, Stray-Gundersen J., Levine BD. Individual variation in response to altitude training. J Appl Physiol 1998; 85(4): 1448-56.

Received: December 12, 2009

Accepted: March 26, 2010

Published: March 31, 2010

Address for correspondence:

Boro Štrumbelj

Faculty of sport

Gortanova 22

Slovenia

e-mail: boro.strumbelj@guest.arnes.si

Anton Ušaj: anton.usaj@fsp.uni-lj.si 